\title{
The Perfect Storm: CNS Drug Development in Trouble
}

\author{
Andrew A. Nierenberg, MD
}

"For every complex problem there is an answer that is clear, simple, and wrong".

H.L. Menken

The high cost and high risk of central nervous system (CNS) drug development coupled with decreased opportunities for pharmaceutical companies to recoup their investments and make profits to further reinvest in research and development (R\&D) is now reaching its logical conclusion: companies will curtail making new medications for CNS diseases. As Tom Insel reports in his National Institute of Mental Health (NIMH) Director's Blog,' GlaxoSmithKline (GSK) and AstraZeneca (AZ) will no longer develop psychiatric medications. In the past, GSK took the risk of studying the anticonvulsant lamotrigine (LTG) for bipolar depression using an unorthodox drug development program. ${ }^{2}$ LTG was subsequently approved for preventing mood episodes in bipolar disorder. ${ }^{3} \mathrm{AZ}$ developed quetiapine (OTP) as an antipsychotic and subsequently found that QTP worked as an antimanic and antidepressant for bipolar disorder. ${ }^{4-6}$ LTG and QTP are now among the most widely prescribed medications in psychiatry. The loss of GSK's and AZ's programs is a major blow to the psychiatric community. Dr. Insel optimistically writes that the NIMH may play a role in further R\&D by finding a few key discoveries and help develop a new pipeline. But he is also cautious about replicating or replacing pharma, and realistically notes that the limited budget of the NIMH will not account for the cost of bringing even one new medication to market. He also states that "Conducting clinical research more efficiently may free up some resources required to make a major investment. But limits in the NIMH's funding clearly indicate that, as we set priorities, hard choices will need to be made between investing in new medications and attempting to optimize the use of existing ones". ${ }^{1}$

Pharma's retreat from CNS drug development, coupled with woefully inadequate funding of the NIMH, does not bode well for the future of psychiatric medication development. With the exception of lithium, I am not aware of a single medication brought to market from sources other than from pharma in the past 35 years (if anyone knows of any, please let me know). Assuming an inflation-adjusted estimate of an NIMH budget of $\$ 35$ billion over 35 years, no new treatments after $\$ 35$ billion is quite impressive. Dr. Insel's dilemma about what to invest in with a limited (and perhaps flat or shrinking) budget presents a formidable problem for the field. If the NIMH invests in new medications at the expense of funding clinical effectiveness research (that provides data for clinicians to make better decisions about prescribing existing treatments) then the changes in treatment will not occur for many years. Today's clinicians need better guidance for today's patients and if the dream of the new health care reform is to be realized, we need those answers for this generation and cannot wait for the next generation. On the other hand, if the NIMH invests in clinical effectiveness research at the expense of developing new medications, then drug development will be further imperiled.

One solution proposed by Dr. Insel is to conduct "clinical research more efficiently". I worry 
that this solution may not be so easy to achieve. Clinical research is currently quite costly because it requires many people to conduct it properly, with precision and integrity, while maintaining the safety of the participants. Clinical research requires a substantial infrastructure and many sites to complete studies that include a sufficient number of participants to answer the study questions. Coordination of sites, researchers, research assistants, data flow, database management, statistical support, and the generation of manuscripts takes time and expertise. Following the Sequenced Treatment Alternatives to Relieve Depression (STAR*D), the Depression Trials Network (DTN) was formed and subsequently conducted several studies that have examined the emergence of suicidal ideation during selective serotonin reuptake inhibitor treatment, effectiveness of combinations of antidepressants and combinations of antidepressants and antipsychotics, and Combining Medication to Enhance Depression Outcomes, a study of combinations of antidepressants versus monotherapy. All of these effectiveness trials used the infrastructure that NIMH had invested in to conduct STAR*D. The studies were started quickly, used expert sites, were coordinated with fidelity to the protocols, used an efficient tablet-based paperless data management system, recruited close to $100 \%$ of the planned number of participants, had an excellent continuous performance improvement system, and were conducted on time and within budget.

For complex reasons, the DTN finished the term of its NIMH contract and was dismantled. By all definitions and performance metrics, the DTN conducted the clinical effectiveness research as efficiently as possible and is unlikely to be recre- ated (or refunded) anytime soon. It is unclear how the DTN could have been more efficient. If the NIMH decides to get out of the clinical trial business, then no other agency is likely to study how to "optimize existing" psychiatric treatments. If available treatments are not optimized, then the diminishing number of studies from pharma will continue to focus on efficacy (differences of active drugs from placebo) and will fail to inform clinical practice. With pharma retreating from drug development, fewer new drugs will make it to market, and those that do will not have the data about how clinicians should optimally use them.

One could easily be discouraged given the withdrawal of pharma from CNS drug development and limited funding from NIMH. The hope is on page 1,617 of the 2010 HealthCare Reform act that establishes a Patient-Centered Outcomes Research Institute for comparative effectiveness research to be funded at $\$ 500$ million/year. It will be essential that psychiatric disorders are included in this important endeavor. Only then will we and our patients survive this perfect storm. CNS

\section{REFERENCES}

1. NIMH Director's Blog. http:/www.nimh.nih.gov/about/director/2010/who-will-developthe-next-generation-of-medications-for-mental-illness.shtml. Accessed: March 30, 2010.

2. Geddes JR, Calabrese JR, Goodwin GM. Lamotrigine for treatment of bipolar depression: independent meta-analysis and meta-regression of individual patient data from five randomised trials. Br J Psychiatry. 2009;194:4-9.

3. Calabrese JR, Bowden CL, Sachs $G$, et al. A placebo-controlled 18-month trial of lamotrigine and lithium maintenance treatment in recently depressed patients with bipolar 1 disorder. J Clin Psychiatry. 2003;64:1013-1024.

4. Bowden $\mathrm{CL}$, Grunze $\mathrm{H}$, Mullen J, et al. A randomized, double-blind, placebo-controlled efficacy and safety study of quetiapine or lithium as monotherapy for mania in bipolar disorder. J Clin Psychiatry. 2005;66:111-121.

5. Calabrese JR, Keck PE, Macfadden W, et al. A randomized, double-blind, placebo-controlled trial of quetiapine in the treatment of bipolar I or II depression. Am J Psychiatry. 2005:162:1351-1360.

6. Thase ME, Macfadden W. Weisler RH, et al. Efficacy of quetiapine monotherapy in bipolar | and II depression: A double-blind, placebo-controlled study (The BOLDER I| studyl. J Clin Psychopharmacol. 2006;26:600-609.

Dr. Nierenberg is professor of psychiatry at Harvard Medical School, co-director of the Bipolar Clinic and Research Program, and associate director of the Depression Clinical and Research Program at Massachusetts General Hospital (MGH) in Boston.

Faculty Disclosures: Dr. Nierenberg consulted to or served on the advisory boards of Abbott, Appliance Computing, Inc., Brain Cells, Inc., BristolMyers Squibb, Eli Lilly, EpiQ, Forest, GlaxoSmithKline, Janssen, Jazz, Merck, Novartis, Pamlab, Pfizer, PGx Health, Pharmaceutica, Schering-Plough, Sepracor, Shire, Somerset, Takeda, and Targacept; he has received research support from Cederroth, Cyberonics, Forest, Medtronics, NARSAD, the NIMH, Ortho-McNeil-Janssen, Pamlab, Pfizer, Shire, and the Stanley Foundation through the Broad Institute; he has received past support from BristolMyers Squibb, Cederroth, Eli Lilly, Forest, GlaxoSmithKline, Janssen, Lictwer Pharma, Pfizer, and Wyeth; he has received honoraria from the MGH Psychiatry Academy (MGHPA activities are supported through Independent Medical Education grants from AstraZeneca, Eli Lilly, and Janssen; he earns fees for editorial functions for CNS Spectrums through MBL Communications, Inc., and Psychiatric Annals through Slack, Inc.; he receives honoraria as a CME Executive Director for the Journal of Clinical Psychiatry through Physicians Postgraduate Press; he has been on the speaker's bureaus of Bristol-Myers Squibb, Cyberonics, Eli Lilly, Forest, GlaxoSmithKline, and Wyeth; he has received royalties from Cambridge University Press and Belvoir Publishing; he owns stock options in Appliance Computing, Inc.; and owns the copyrights to the Clinical Positive Affect Scale and the MGH Structured Clinical Interview for the Montgomery Asberg Depression Scale, exclusively licensed to the MGH Clinical Trials Network and Institute. 